\title{
THE SECOND DUAL OF THE SPACE OF CONTINUOUS FUNCTIONS. III
}

\author{
BY \\ SAMUEL KAPLAN(')
}

1. Introduction. In this paper we turn our attention to a locally compact space $X$. There are several distinct spaces of continuous functions on $X$ which have been objects of study; in particular, the space $C$ of all continuous functions, the space $C^{b}$ of bounded ones, the space $C^{\omega}$ of those vanishing at infinity, and the space $C^{k}$ of those with compact support. It is the second duals of the last two which will interest us here.

$C^{\omega}$ and its first and second duals, $L^{\omega}, M^{\omega}$, are Banach lattices, and their study offers little over the case of compact $X$. Thus we may expect that the new results are to be found in the study of $C^{k}$ and its first and second duals, $L^{k}, M^{k}$, and in their relations to $C^{\omega}, L^{\omega}$, and $M^{\omega}$. For example, while $L^{\omega}$ is the space of bounded Radon measures on $X$, the unbounded ones are to be found in $L^{k}$.

As in our previous papers $[8 ; 9]$, we attempt as far as possible to use vector lattice techniques, considering them as the natural tools in this subject and topological tools as necessary evils at best. Thus for example $L^{k}$ is defined as the vector lattice of all bounded linear functionals on $C^{k}$. The definition of $M^{k}$ is not so obvious, and we postpone its discussion to $\S 3$.

$\$ 2$ is devoted to various theorems which we will need about ideals in vector lattices. In $\$ \S 3$ and 4 we establish the basic properties of our six spaces, and the elementary relations between them. A study of these spaces has already been initiated by J. E. Mack in his doctoral dissertation [10], and the material of $\S 3$ is taken from this dissertation. Mack proceeds to a study of $M^{\omega}$ and $M^{k}$ along lines similar to those followed in $[8 ; 9]$. We, however, are essentially brought to a stop by a basic question on the structure of $M^{k}$, and we devote most of the remainder of the paper to this question-without succeeding in answering it.

One formulation of the question is as follows. $C^{k}$ has the property that each of its elements has a relative strong order unit in $C^{k}$, that is, given $f \in C^{k}$, there exists $g \in C^{k}$ such that every element of the closed ideal generated by $f$ is dominated by some multiple of $g$. Our question is: Does $M^{k}$ have this same property?

In $\S 5$, we examine $C$ as a ring of operators on $C^{k}, L^{k}$, and $M^{k}$. In $\S 6$, we

Presented to the Society, February 16, 1961 ; received by the editors February 8, 1961.

(1) The work on this paper was supported by National Science Foundation Grants NSFG8814 and NSF-G12983, and aided by residence during the summer of 1960 as a member of the Institute for Advanced Study. 
examine the (locally compact) Stone space of $M^{k}$. Our above question can then be formulated as follows: Does every element of $M^{k}$, considered as a continuous function on this Stone space, have compact support? The best that we can conclude (following an argument of Mack) is that every element of $M^{k}$ has pseudocompact support.

Added May, 1961. I have been informed by J. E. Mack that he has an example which answers this question in the negative; it will appear in a paper he is preparing for publication. We are letting the present paper stand as it is, since the material here is of independent interest and clears groundwork for a continued study of $M^{k}$. Note that with the existence of Mack's example, (7.8) below takes on more significance. There also remains the question raised after the proof of (3.5).

2. Ideals. We assume a knowledge of our first two papers $[8 ; 9]$. We emphasize that by convergence we mean that defined by the order $[9, \S 3]$, and unless otherwise stated, terms such as continuous, closure, dense, etc. are with respect to this convergence.

Given a vector lattice $E$, the vector lattice $\Omega(E)$ of bounded linear functionals on $E$ and its closed ideal $\tilde{\Omega}(E)$ of continuous ones will of ten be written simply $\Omega$ and $\tilde{\Omega}$. If $A$ is a subset of $E, A^{\perp}$ will denote its annihilator in $\Omega$.

If an ideal $I$ in $E$ is a direct summand of $E$, we have a natural projection of $E$ onto $I$. We denote the image of a set $A$ of $E$ under this projection by $A_{I}$; in particular the component in $I$ of an element $b$ of $E$ is denoted by $b_{I}$.

Now let $I$ be any ideal in $E$. There are three vector lattices associated with $I$ in a natural way. For one, the quotient vector space $E / I$ is a vector lattice under the order defined by taking for the positive cone the image of $E_{+}$in $E / I$ [4, Chapter II, $\S 1$, Ex. 4$]$; by $E / I$ we will mean this vector lattice. The other two vector lattices are the closed ideals $I^{\perp}$ and $I^{\perp \prime}$ in $\Omega$. We have of course $\Omega=I^{\perp} \oplus I^{\perp \prime}$. It is immediate that $\Omega(E / I)=I^{\perp} . \Omega(I)$, on the contrary, cannot in general be identified with a subspace or quotient space of $\Omega$. Nevertheless, there are close relations between $\Omega(I)$ and $\Omega$, or more specifically, between $\Omega(I)$ and $I^{\perp \prime}$; and we proceed to examine some of these.

First, a remark on $I^{\perp \prime}$. An element $\phi$ of $I^{\perp \prime}$ is completely determined by its values on $I$ : for, suppose $\phi(I)=0$; then $\phi \in I^{\perp}$, and since $I^{\perp \prime} \cap I^{\perp}=0$, we have $\phi=0$. It follows that given $\phi \in I^{\perp \prime}$, its values on all of $E$ should be obtainable from its values on $I$. The specific procedure for doing this is described by

(2.1) Given $\phi \in I^{\perp \prime}, \phi \geqq 0$, and $a \in E_{+}$, then $\phi(a)=\sup _{c \in I, 0 \leq c \leq a} \phi(c)$.

Proof. Denote the above supremum by $\rho(a) .0 \leqq \rho(a) \leqq \phi(a)<\infty$; hence, letting $a$ vary, we have a non-negative function $\rho$ defined on $E_{+}$. Straightforward computation gives us that $\rho(a+b)=\rho(a)+\rho(b)$ for all $a \in E_{+}, b \in E_{+}$. It follows [4, Chapter II, $\$ 1$, Proposition 2] that $\rho$ can be extended to a unique element of $\Omega$. Since $0 \leqq \rho \leqq \phi$, it lies in $I^{\perp \prime}$; since it agrees with $\phi$ on $I$, it is identical with $\phi$. This completes the proof. 
We turn to $\Omega(I)$. The natural mapping $\pi: \Omega \rightarrow \Omega(I)$ defined by $\pi \phi=\phi \mid I$ is an order-preserving linear mapping of $\Omega$ onto an ideal of $\Omega(I)[9,(2.4)]$. It is easily shown that the restriction of $\pi$ to $I^{\perp \prime}$ is an isomorphism of $I^{\perp \prime}$ onto this ideal. We will identify the two, and can thus write

(2.2) $I^{\perp \prime}=\Omega(I) \cap \Omega$ and is an ideal in $\Omega(I)$.

With this identification, the question, "can a given element $\phi$ of $\Omega(I)$ be extended to all of $E$ ?" becomes simply, "does $\phi$ lie in $\Omega$ also?" We have

(2.3) Given $\phi \in \Omega(I)_{+}$, a necessary and sufficient condition that $\phi$ lie in $\Omega$ is that for every $a \in E_{+}, \sup _{c \in I}$, 0scsa $\phi(c)<+\infty$.

Proof. If this condition is satisfied, the procedure used in the proof of (2.1) gives us an element $\rho$ of $I^{\perp \prime}$ which agrees with $\phi$ on $I$, hence is identical with $\phi$. The necessity is clear.

In general, $I^{\perp \prime}$ need be neither dense nor closed in $\Omega(I)$. Otherwise stated, if we denote the closure of $I^{\perp \prime}$ in $\Omega(I)$ by $H$ and write $\Omega(I)=H \oplus H^{\prime}$, then in general $H^{\prime} \neq 0$ and $I^{\perp \prime} \neq H$. There are various sufficient conditions on $I$, under which $I^{\perp \prime}$ has one or both of these properties. One obvious one is that $I$ is a direct summand of $E$; then $I^{\perp \prime}=\Omega(I)$. Another condition, which will actually be satisfied by some of the spaces in which we are interested, is given in

(2.4) Suppose I has the following property: every countable subset of I which is bounded in $E$ is already bounded in $I$. Then $I^{1 \prime}=\Omega(I)$.

Proof. Consider $\phi \in \Omega(I)_{+}$. We show that $\phi$ satisfies the condition of (2.3). Suppose not, that is, there exists $a \in E_{+}$such that $\sup _{c \in I, 0 \leq c s a} \phi(c)=+\infty$. Choose a sequence $\left\{c_{n}\right\}, c_{n} \in I, 0 \leqq c_{n} \leqq a$ such that $\phi\left(c_{n}\right) \geqq n, n=1,2, \cdots$. But by our hypothesis on $I,\left\{c_{n}\right\}$ is bounded above in $I$, hence $\left\{\phi\left(c_{n}\right)\right\}$ must be bounded.

Corollary. If $E$ is $\sigma$-complete and $I$ is $\sigma$-closed in $E$, then $I^{1 \prime}=\Omega(I)$.

Some notation. Consider a fixed element $a$ of $E$, and let $J$ be the ideal generated by $a$. If the closure $\bar{J}$ of $J$ is a direct summand of $E$, we will denote the projection in $\bar{J}$ of a set $A$ by $A_{a}$ instead of $A_{\bar{J}}$, and the component in $\bar{J}$ of an element $b$ by $b_{a}$ instead of $b_{\bar{J}}$. Thus in particular, $\bar{J}=E_{a}$. Moreover, whatever the relation of $\bar{J}$ to $E, J^{\perp \prime}$ is always a direct summand of $\Omega$; we will denote the projection in $J^{\perp \prime}$ of a subset $A$ of $\Omega$ by $A_{a}$ also (instead of $A_{J}{ }^{\prime \prime}$ ), and the component in $J^{\perp \prime}$ of an element $\phi$ of $\Omega$ by $\phi_{a}$ (instead of $\phi_{J^{\perp}}$ ). In particular, $J^{\perp \prime}=\Omega_{a}$.

The following is easily shown.

(2.5) For each $\phi \in \Omega_{+}, \phi=\bigvee_{a \in B} \phi_{a}$.

An equivalent formulation of this is that $\phi_{a} \uparrow \phi$ as $a$ runs through the elements of $E_{+}$(which is a directed set under its order). A third formulation 
is that the ideal $\bigcup_{a \in E} \Omega_{a}\left(=\sum_{a \in E} \Omega_{a}\right)$ is dense in $\Omega$.

Returning to $\Omega(I)$, we will also need the following.

(2.6) Suppose the condition of (2.4) holds only for countable sets which lie in principal ideals of $I$. Then $I^{1 \prime}$ is dense in $\Omega(I)$.

Proof. From (2.5), it is enough to show that for each $a \in I, \Omega(I)_{a} \subset \Omega$. This follows by the same argument as was used in (2.4).

A natural question is: if $I$ is dense in $E$, can we say anything about the relation of $I^{\perp \prime}$ to either $\Omega$ or $\Omega(I)$ ? The answer is no, in general. However, as would be expected, we can draw conclusions about its relation to $\tilde{\Omega}$ and $\tilde{\Omega}(I)$. First,

(2.7) If $I$ is dense in $E$, then $\tilde{\Omega} \subset \bar{\Omega}(I)$.

Proof. Consider $\phi \in \tilde{\Omega}$. Since $\tilde{\Omega}$ is an ideal, $\phi_{I^{\perp}} \in \tilde{\Omega}$ also; it therefore follows from $\phi_{I^{\perp}}(I)=0$ that $\phi_{I} \perp(E)=0$, and thus $\phi_{I^{\perp}}{ }^{\perp}=0$. This gives us $\phi \in I^{\perp \prime} \subset \Omega(I)$. Since the continuity of $\phi$ on $E$ implies its continuity on $I$, we have finally that $\phi \in \tilde{\Omega}(I)$.

(2.7) together with $[9,(3.5)]$ gives us

(2.8) Suppose $E$ is complete and $\tilde{\Omega}$ is separating on $E$. If $I$ is a dense ideal in $E$, then $\tilde{\Omega}$ is a dense ideal in $\dot{\Omega}(I)$.

Note that contained in this conclusion is the statement that $\tilde{\Omega}(I) \cap \Omega=\tilde{\Omega}$.

We close this section with a definition we will need later. An element $a$ of $E$ will be called a relative strong order unit for an ideal $I$ if every element of $I$ is dominated by some multiple of $a$.

3. The first and second duals. The material in this section is from Mack's thesis [10]. However we develop it in our own way and with our own notation. We confine ourselves entirely to real functions. For any compact (Hausdorff) space $Z$, we will denote the Banach lattice of continuous functions on $Z$, and its first and second duals, by $C(Z), L(Z)$, and $M(Z)$ respectively.

In the present paper, $X$ will be a fixed locally compact (Hausdorff) space. We will use the following notation:

$C$ : the set of all continuous functions on $X$.

$C^{\omega}$ : the set of all continuous functions on $X$ which vanish at infinity.

$C^{k}$ : the set of all continuous functions on $X$ which have compact support.

$C^{\omega}$ will be treated as a vector lattice, and $C^{k}$ as an ideal in it. $C$, on the contrary, will appear as a ring of operators on $C^{k}$ and on its first and second duals. $C^{\omega}$ and $C^{k}$ and their first and second duals are intimately related, and will be studied together. First, however, we collect some elementary properties of $C^{\omega}$. This is most expeditiously accomplished by imbedding $X$ in its Alexandroff one-point compactification $\alpha X$. We denote the new point by $\bar{x}$. $C^{\omega}$ is the ideal in $C(\alpha X)$ consisting of all the elements vanishing at $\bar{x}$. It is 
norm-closed, hence is itself a Banach lattice. Denoting the constant function on $\alpha X$ of value 1 by $1(\alpha X)$, we have $C(\alpha X)=C^{\omega}+R \cdot 1(\alpha X)(R \cdot 1(\alpha X)$ is the one-dimensional linear subspace generated by $1(\alpha X))$. We note also that $f \wedge 1(\alpha X) \in C^{\omega}$ whenever $f \in C^{\omega}$.

We denote $\Omega\left(C^{\omega}\right)$ by $L^{\omega}$ and $\Omega\left(L^{\omega}\right)$ by $M^{\omega}$. Their relations to $L(\alpha X)$ and $M(\alpha X)$ are quite simple. For, writing $L(\alpha X)=\left(C^{\omega}\right)^{\perp} \oplus\left(C^{\omega}\right)^{\perp \prime}$, and noting that $C^{\omega}$ has co-dimension one in $C(\alpha X)$, we obtain that $\left(C^{\omega}\right)^{\perp}$ is one-dimensional, hence is precisely $R \bar{x}$. Since moreover, by standard Banach space theory, $\left(C^{\omega}\right)^{\perp \prime}$ can be identified with the dual of $C^{\omega}$, we have $L(\alpha X)=L^{\omega} \oplus R \bar{x}$. This decomposition gives us in turn the decomposition $M(\alpha X)=M^{\omega} \oplus\left(L^{\omega}\right)^{\perp}$. If we denote the element of $M(\alpha X)_{0}[8, \S 5]$ which has value 1 on $\bar{x}$ and 0 on all $x \in X$ by $\bar{e}$, this becomes $M(\alpha X)=M^{\omega} \oplus R \bar{e}$. As we know [9, (4.1)], $M(\alpha X)$ $=\tilde{\Omega}(L(\alpha X))$; it follows easily that $M^{\omega}=\tilde{\Omega}\left(L^{\omega}\right)$. In summary,

(3.1) $L(\alpha X)=L^{\omega} \oplus R \bar{x}, M(\alpha X)=M^{\omega} \oplus R \bar{e}$, and $M^{\omega}=\tilde{\Omega}\left(L^{\omega}\right)$.

$1(\alpha X)$ is the strong order unit for $M(\alpha X)(\|f\| \leqq 1$ if and only if $|f| \leqq 1(\alpha X))$, hence its component $1(\alpha X)_{M^{\omega}}$ is the strong order unit for $M^{\omega}$. It is this component which will henceforth be denoted by 1 . We should be careful to note that $1 \notin C^{\omega}$. However, it is easily shown that $f \wedge 1 \in C^{\omega}$ whenever $f \in C^{\omega}$; also that for every $\mu \in L^{\omega},\|\mu\|=1(\mu)$.

We turn to $C^{k}$ and its duals. $C^{k}$ is an ideal in $C^{\omega}$. It has, in addition, three basic properties:

(3.2) $C^{k}$ is norm-dense in $C^{\omega}$.

(3.3) For each $f \in C^{k}$, if a subset of the closed ideal in $C^{k}$ generated by $f$ is bounded in $C^{\omega}$, then it is already bounded in $C^{k}$.

(3.4) For each $f \in C^{k}$, the closed ideal in $C^{k}$ generated by $f$ has a relative strong order unit in $C^{k}$ (cf. the definition at the end of $\$ 2$ ).

We denote $\Omega\left(C^{k}\right)$ by $L^{k}$. Since $C^{k}$ is an ideal in $C^{\omega}$, we can write $L^{\omega}=\left(C^{k}\right)^{\perp} \oplus\left(C^{k}\right)^{\perp \prime}$. However property (3.2) gives us that $\left(C^{k}\right)^{\perp}=0$, hence $L^{\omega}=\left(C^{k}\right)^{\perp \prime}$, and we thus have $L^{\omega} C L^{k}$. In fact,

(3.5) $L^{\omega}$ is a dense ideal in $L^{k}$. It consists of those elements $\mu$ of $L^{k}$ satisfying $\sup _{h \in C^{k}, 0 \leq h \leq 1(\alpha X)}|\mu|(h)<\infty$.

Proof. The first statement follows from the above equality combined with (2.6) and (3.3). We show the second. The necessity of the condition is trivial. The sufficiency follows from (2.3) and the fact that each element of $C^{\omega}$ is dominated by a multiple of $1(\alpha X)$.

The elements of $L^{k}$ are the Radon measures on $X$; those of $L^{\omega}$ are the bounded Radon measures.

There is a natural question which can be raised at this point. $X$ is a subset of $L^{\omega}$; we denote by $L^{\omega}{ }_{0}$ and $L^{k_{0}}$ the closed ideals generated by $X$ in $L^{\omega}$ and 
$L^{k}$ respectively. The elements of $L^{k}{ }_{0}$ are the atomic (Radon) measures on $X$ and those of $L \omega_{0}$, the bounded atomic (Radon) measures on $X[5, \S 5$, no. 10]. The question is: if every atomic measure on $X$ is bounded, does it follow that every measure is bounded? We do not know the answer to this question. It seems to us to be related to the question which we stated in the introduction, and which we will examine later.

Now let's consider $\Omega\left(L^{k}\right)$. Since $L^{\omega}$ is an ideal in $L^{k}$, we can write $\Omega\left(L^{k}\right)$ $=\left(L^{\omega}\right)^{\perp \prime} \oplus\left(L^{\omega}\right)^{\perp}$, with $\left(L^{\omega}\right)^{\perp \prime}=M^{\omega} \cap \Omega\left(L^{k}\right)$. But $M^{\omega}=\tilde{\Omega}\left(L^{\omega}\right)$, hence from the remark following (2.8), $\left(L^{\omega}\right)^{\perp \prime}=\tilde{\Omega}\left(L^{k}\right)$, and we have $\Omega\left(L^{k}\right)=\tilde{\Omega}\left(L^{k}\right) \oplus\left(L^{\omega}\right)^{\perp}$.

It is $\tilde{\Omega}\left(L^{k}\right)$ - not $\Omega\left(L^{k}\right)$-which we will denote by $M^{k}$. Our reasons are the following. In the first place, we already have $M(\alpha X)=\tilde{\Omega}(L(\alpha X))$ and $M^{*}$ $=\tilde{\Omega}\left(L^{\omega}\right)$. Secondly, it is only the elements of $\tilde{\Omega}\left(L^{k}\right)$ which are "accessible" to $C^{k}$ by convergence $[9,(3.10)]$ and by the various vector lattice topologies on $\Omega\left(L^{k}\right)$ (almost as though the rest of $\Omega\left(L^{k}\right)$ can never be "known" starting from the original space $C^{k}$ ). Finally, there is even a question as to the very existence of any elements in $\Omega\left(L^{k}\right)$ outside of $\tilde{\Omega}\left(L^{k}\right)$; Mack [10] has pointed out that if $X$ is discrete, the existence of such elements is equivalent to Mackey's vector lattice formulation of Ulam's problem on the existence of inaccessible cardinals.

Summing up,

(a) $M^{k}=\tilde{\Omega}\left(L^{k}\right)=M^{\omega} \cap \Omega\left(L^{k}\right)$;

(3.6) (b) $M^{k}$ is a dense ideal in $M^{\omega}$ (cf. (2.8));

(c) $\Omega\left(L^{k}\right)=M^{k} \oplus\left(L^{\omega}\right)^{\perp}$.

Also,

(3.7) The closure of $C^{k}$ in $M^{\omega}$ is $M^{\omega}$.

This follows from $[9,(3.10)]$ and (b) above.

4. Additional structure. Throughout this paper, unless otherwise stated, the letter $K$ will always denote a compact subset of $X$. Consider such a subset $K . C(K)$ cannot in general be identified with a subset of $C^{k}$. However $L(K)$ and $M(K)$ are direct summands of $L^{k}$ and $M^{k}$ respectively. For, let $I$ be the ideal $I=\left\{f \in C^{k} \mid f(x)=0\right.$ on $\left.K\right\}$; by the Tietze extension theorem, $C(K)$ is isomorphic with $C^{k} / I$. It follows $L(K)=I^{\perp}$, and thus we can write $L^{k}$ $=L(K) \oplus I^{\perp \prime}$ and $M^{k}=M(K) \oplus\left(L(K)^{\perp}-i n-M^{k}\right)$.

We note that the fact that $C(K)$ is not a subspace of $C^{k}$ is now partly corrected, since its natural imbedding in $M(K)$ makes it a subspace of $M^{k}$. A word of emphasis: suppose $f$ is a continuous function on $X$ with support in $K$; then $f$ can be considered both as an element of $C^{k}$ and as an element of $C(K)$; in $M^{k}$, these are the same element.

We proceed to examine the $L(K)$ 's and $M(K)$ 's.

(4.1) For every $K, L(K) \subset L^{\omega}$. 
Proof. Consider $\mu \in L(K)_{+}$. Given any $f \in C^{\omega}$, we can write $f=g+h$, where $g \in C^{k}$ and $h$ vanishes on $K$. Thus $h$ lies in the ideal $I$ we defined above, hence $\mu(h)=0$ and we have $\mu(f)=\mu(g)$. It follows easily that $\mu$ satisfies the condition of (2.3).

(4.2) The set $\bigcup_{\mathrm{a} 11 K} L(K)$ is an ideal in $L^{\omega}$, and is dense in $L^{k}$.

That it is an ideal is shown by the identity

$$
\bigcup_{\text {all } K} L(K)=\sum_{\text {all } K} L(K) \text {. }
$$

That it lies in $L^{\omega}$ follows from (4.1). Finally, given any $f \in C^{k}$, let $K$ be its support. Then $L^{k} f C L(K)$ (cf. the discussion preceding (2.5) for the notation $\left.L_{f}^{k}\right)$, and (2.5) gives us the last statement.

Similarly,

(4.3) The set $\mathrm{U}_{\mathrm{all} K} M(K)$ is an ideal in $M^{k}$, and contains $C^{k}$ (hence is dense in $\left.M^{\omega}\right)$.

As we know $[9,(3.9)], L^{k}=\tilde{\Omega}\left(M^{k}\right)$. We show that also

(4.4) $\tilde{\Omega}\left(\bigcup_{\mathrm{a} 11 K} M(K)\right)=L^{k}$.

Proof. That $L^{k} \subset \tilde{\Omega}\left(\bigcup_{a 11} K M(K)\right)$ follows from (2.7). Conversely, consider $\phi \in \tilde{\Omega}\left(\mathrm{U}_{\mathrm{all} K} M(K)\right) . \phi$ restricted to $C^{k}$ is a bounded linear functional on $C^{k}$; otherwise stated, $\phi$ coincides on $C^{k}$ with some $\mu \in L^{k}$. Since the closure of $C^{k}$ in $\mathrm{U}_{\mathrm{al1} K} M(K)$ is $\mathrm{U}_{\mathrm{al1} K} M(K)$, the continuity of both $\phi$ and $\mu$ on $\mathrm{U}_{\mathrm{al1} K} M(K)$ implies they coincide on all of it.

There is another subspace of $L^{k}$ in which we will be interested. This is the $\sigma$-closure of $L^{\omega}$. We denote it by $\sigma L^{\omega}$. The ideal $\bigcup_{\mathrm{a} 11 K} L(K)$ is also $\sigma$-dense in $\sigma L^{\omega}$. For, it is dense in $L^{\omega}$, hence norm-dense there ( $L^{\omega}$ is an $(L)$-space), hence $\sigma$-dense there; hence its $\sigma$-closure in $L^{k}$ coincides with that of $L^{\omega}$. If $X$ is $\sigma$-compact, $\sigma L^{\omega}=L^{k}$. In general, however, it is a proper ideal.

(4.5) $\tilde{\Omega}\left(\sigma L^{\omega}\right)=M^{k}$.

Proof. We note first that

$$
M^{k}=\tilde{\Omega}\left(L^{k}\right) \subset \tilde{\Omega}\left(\sigma L^{\omega}\right) \subset \tilde{\Omega}^{\sigma}\left(\sigma L^{\omega}\right) \subset \Omega^{\sigma}\left(L^{\omega}\right)=M^{\omega} .
$$

(We recall that $\Omega^{\sigma}(E)$ is the set of elements of $\Omega(E)$ which are $\sigma$-continuous on $E[9,(5.3)]$.) The only new relation in this chain is that $\Omega^{\sigma}\left(\sigma L^{\omega}\right) C \Omega^{\sigma}\left(L^{\omega}\right)$. This can be proved by the same kind of argument as was used in (2.7). Now the corollary of (2.4) gives us that $\Omega\left(\sigma L^{\omega}\right) \subset \Omega\left(L^{k}\right)$. Combining this with the last part of (i), $\Omega^{\sigma}\left(\sigma L^{\omega}\right) \subset M^{\omega} \cap \Omega\left(L^{k}\right)=M^{k}$. Thus, from (i) again, $M^{k} \subset \Omega^{\sigma}\left(\sigma L^{\omega}\right)$ $C M^{k}$, and we have the desired equality.

REMARK. We have also showed here that $\bar{\Omega}\left(\sigma L^{\omega}\right)=\Omega^{\sigma}\left(\sigma L^{\omega}\right)$.

The spaces of common interest in integration are related to our spaces as 
follows. Let $\mu$ be an element of $L^{k}$. In accordance with our general notation, we denote the closed ideal in $L^{k}$ generated by $\mu$ by $L^{k}{ }_{\mu}$, the ideal $L^{k}{ }_{\mu} \cap L^{\omega}$ (closed in $L^{\omega}$ ) by $L^{\omega}{ }_{\mu}$, and their dual ideals in $M^{k}, M^{\omega}$ by $M^{k}{ }_{\mu}, M^{\omega_{\mu}}$ respectively (i.e. $M^{k}{ }_{\mu}=\left(L^{k}{ }_{\mu}\right)^{\perp \prime}-i n-M^{k}$ and $\left.M^{\omega_{\mu}}=\left(L^{\omega_{\mu}}\right)^{\perp \prime}-i n-M^{\omega}\right)$. $L^{\omega_{\mu}}$ and $M^{\omega_{\mu}}$ can be identified, in the Bourbaki notation, with $L^{1}(\mu)$ and $L^{\infty}(\mu)[4$, Chapter IV]; and $L^{k}{ }_{\mu}$, with the space of locally $\mu$-integrable functions modulo the locally $\mu$-negligible ones [5].

The following is immediate.

(4.6) (a) $L^{k}{ }_{\mu}$ and $M^{k}{ }_{\mu}$ are each other's duals with respect to continuity; and similarly for $L^{\omega_{\mu}}$ and $M^{\omega_{\mu}}$.

(b) $L^{\omega_{\mu}}$ is a dense ideal in $L^{k}{ }_{\mu}$; and $M^{k}{ }_{\mu}$ is a dense ideal in $M^{\omega_{\mu}}$.

(c) $M^{\omega}{ }_{\mu}$ is the completion of $C^{k}{ }_{\mu}$ under $|w|\left(C^{k}{ }_{\mu}, L^{\omega_{\mu}}\right)$; and $M^{k}{ }_{\mu}$ is its completion under $|w|\left(C^{k}{ }_{\mu}, L^{k}{ }_{\mu}\right)$.

5. The Question. In his thesis, Mack initiates a study of $M^{k}$ and $M^{*}$ along the lines of our previous papers $[8 ; 9]$. Our own attention, however, will be absorbed by the unanswered question mentioned in the introduction. The work in the remainder of this paper, while of interest in itself, has been largely motivated by the above question. We will henceforth refer to it as the Question. Before formulating it, we state two properties which we will use. They are based on elementary properties of complete vector lattices, and we do not stop to prove them.

(5.1) (a) For each $f \in M^{k}$ and $\lambda>0, M_{(f-\lambda 1)^{+}}^{k}=M^{\omega}{ }_{(f-\lambda 1)+}$ (otherwise stated, $M^{k}(f-\lambda 1)+i s$ closed in $\left.M^{\omega}\right)$.

(b) For each $f \in M^{k}, 1_{(n f-1)+} \uparrow 1_{f}$ as $n \rightarrow \infty$.

We now give the

(5.2) First form of the Question: for each $f \in M^{k}$, do we have $1_{f} \in M^{k}$ ?

This first form can be phrased in various ways:

(5.3) For each $f \in M^{k}$, the following are equivalent:

(1) $1_{f} \in M^{k}$;

(2) $M^{k}$ is closed in $M^{\omega}$;

(3) $M^{k_{f}}=M^{\omega_{f}}$;

(4) there exists $g \in M^{k}$ such that $f \in M_{(0-1)^{+}}^{k}$

(5) there exists $g \in M^{k}$ such that $f \wedge(g-1)^{-}=0$;

(6) there exists $g \in M^{k}$ which is a relative strong order unit for $M_{\omega_{f}}$;

(7) there exists $g \in M^{k}$ such that $M^{\omega_{f}} \subset M^{k}{ }_{0}$.

Proof. That (1), (2), (3) are equivalent is clear. To show (1) implies (4), take $g=2 \cdot 1_{f}$, whence $(g-1)^{+}=1_{f}$. We next show (4) implies (3). $M_{f}^{k}$ is closed in $M^{k}$, hence in $M^{k}{ }_{(0-1)+}$, from (4); but this latter is closed in $M^{\omega}$, from (5.1). To show (1) implies (5), take $g=1_{f}$. For the converse implication, note first 
that $1_{f} \wedge 1_{(0-1)^{-}}=0$ and $1-1_{(0-1)^{-}} \leqq g ;$ combining these, we obtain $1_{f} \leqq 1-1_{(0-1)} \leqq g$. To show (1) implies (6), take $g=1_{f}$. It is clear that (6) implies (7) and that (7) implies (1).

REMARK. (5) is the form of the above property singled out by Bauer $[1 ; 2]$. In his terminology, the Question becomes: does $\mathbb{R}^{0}$ coincide with $\mathbb{R}$ for the case $R=M^{k}$ ?

(5.4) Second form of the Question: for each $f \in M^{k}$, does there exist $g \in M^{k}$ which is a relative strong order unit for $M_{f}^{k}$ ?

This second form of the Question is a priori weaker than the first form, as a comparison with (6) in (5.3) above shows. We will prove at the end of 87 that it is actually equivalent to the first form. It has the advantage over the first form of being about an intrinsic property of $M^{k}$.

Before stating the third form of the Question, we show

(5.5) For every $f \in M^{k}, L_{f}^{k} \subset \sigma L^{\omega}$.

Proof. We can assume $f \geqq 0$. From $(5.1), M_{(n f-1)^{+}}^{k}=M_{(n f-1)^{+}}$; hence $L^{k}{ }_{(n f-1)^{+}}=L^{\omega}{ }_{(n f-1)^{+}}$for $n=1,2, \cdots$. Thus it is enough to show that $U_{n} L^{k}{ }_{(n f-1)^{+}}$ is dense in $L^{k}$. This follows from (b) in (5.1) and the easily shown

Lemma. Given a vector lattice $E$ and a collection of ideals $\left\{I_{\alpha}\right\}$, if $\sum_{\alpha} I_{\alpha}$ is dense in $E$, then $\tilde{\Omega} \cap \sum_{\alpha}\left(I_{\alpha}\right)^{\perp \prime}$ is dense in $\tilde{\Omega}$.

(5.6) Third form of the Question: for each $f \in M^{k}$, do we have $L_{f}^{k} \subset L^{\omega}$ ?

It is clear that this form is equivalent to the first form. We will state a fourth form later (7.2).

6. Operators. A linear transformation of a vector lattice $E$ into itself will be called a bounded operator if it carries bounded sets into bounded sets. Positive operators-those linear transformations which carry $E_{+}$into itselfare bounded, and the set of all bounded operators on $E$ forms a partially ordered algebra $B(E)$ under the order defined by taking the positive operators for the positive cone $B(E)_{+}[3]$. The order in $B(E)$ will be denoted by $\leqq$; thus in particular, $T \geqq 0$ means $T$ is a positive operator.

The elements of the subalgebra generated by $B(E)_{+}$will be called real operators. Every real operator is a difference of two positive operators. By a paraphrase of the argument for bounded linear functionals $[4$, Chapter II, $\S 2$, Théorème 1], it can be shown that if $E$ is complete, $B(E)$ is a complete lattice-ordered ring (thus every element is real).

A bounded operator which carries every closed ideal into itself will be called a multiplication operator. The multiplication operators constitute a subalgebra of $B(E)$. If moreover $E$ is complete, they also constitute a closed vector lattice ideal of $B(E)$.

A bounded operator $T$ which preserves convergence in $E$-that is, $a_{\alpha} \rightarrow a$ implies $T a_{\alpha} \rightarrow T a$-will be called a continuous operator. Our interest in this 
paper lies in the continuous multiplication operators on a complete vector lattice $E$. These also constitute a closed vector lattice ideal in $B(E)$. Of more importance, they are a special case of Nakano's dilatators [11], and we can apply his theory. Thus, for example, they commute with each other.

REMARK. Another definition of continuity for a bounded operator $T(E$ assumed complete) is: $a_{\alpha} \downarrow 0$ implies $|T| a_{\alpha} \downarrow 0$. If $T$ is continuous in this sense, then it is continuous in the sense defined above; and the two definitions are equivalent for a multiplication operator.

If $T$ is a bounded operator on a vector lattice $E$, then it is easy to show that the identity $\left(T^{t} \phi\right)(a)=\phi(T a)$ defines a linear transformation of $\Omega$ into itself, and that this linear transformation is uniquely determined by $T . T^{t}$ is called the adjoint (or transpose) of $T$. We of course have all the elementary properties: $(T+S)^{t}=T^{t}+S^{t} ;(\lambda T)^{t}=\lambda T^{t} ;(T S)^{t}=S^{t} T^{t} ;$ if $T$ is the identity map on $E, T^{t}$ is the identity map on $\Omega$; if $T=0, T^{t}=0$, and conversely if $\Omega$ is separating on $E$. In addition, it is easily seen that

(6.1) If $T \geqq 0$, then $T^{t} \geqq 0$; and conversely if $\Omega$ is separating on $E$.

(6.2) If $T^{t}$ is a bounded operator, then it is continuous.

Proof. We can take $T^{t} \geqq 0$. Suppose $\phi_{\alpha} \downarrow 0$ in $\Omega$. Then $T^{t} \phi_{\alpha} \downarrow$; we show $\bigwedge_{\alpha} T^{t} \phi_{\alpha}=0$, or equivalently $[8,(2.2)], \inf _{\alpha}\left[\left(T^{t} \phi_{\alpha}\right)(a)\right]=0$ for every $a \in E_{+}$. This follows from $\left(T^{t} \phi_{\alpha}\right)(a)=\phi_{\alpha}(T a)$ and the supposition that $\phi_{\alpha} \downarrow 0$.

Combining (6.1) and (6.2), we have

CoRollary. If $T$ is real-hence in particular, if $E$ is complete and $T$ a bounded operator-then $T^{t}$ is a continuous operator.

(6.3) If $T$ is a continuous operator, then $T^{i} \tilde{\Omega} \subset \tilde{\Omega}$.

Proof. Consider $\phi \in \bar{\Omega}$. If $a_{\alpha} \rightarrow a$ in $E$, then $T a_{\alpha} \rightarrow T a$, hence $\left(T^{t} \phi\right)\left(a_{\alpha}\right)$ $=\phi\left(T a_{\alpha}\right) \rightarrow \phi(T a)=\left(T^{\prime} \phi\right)(a)$. Thus $T^{\prime} \phi \in \tilde{\Omega}$.

Thus if $T$ is a continuous operator on $E$, the restriction of $T^{t}$ to $\bar{\Omega}$ is a linear transformation of $\tilde{\Omega}$ into itself; it will be called the adjoint (or transpose) of $T$ in $\tilde{\Omega}$.

We will need the following which is essentially contained in Nakano [11, p. 302].

(6.4) Suppose $E$ is reflexive with respect to continuity (that is, $E=\tilde{\Omega}(\tilde{\Omega})$ ). Then the lattice-ordered ring of continuous multiplication operators on $E$ is isomorphic, under the mapping $T \rightarrow T^{t}$, to that on $\dot{\Omega}$.

Proof. Let $T$ be a continuous multiplication operator on $E$. Since $E$ is complete, $T$ is real; hence from the corollary to (6.2) and from (6.3), $T^{t}$ is a continuous operator on $\tilde{\Omega}$. Let $I$ be a closed ideal in $\tilde{\Omega}$ and $\phi \in I$. We show $T^{t} \phi \in I$. In the duality between $\tilde{\Omega}$ and $E$, we have $I=I^{\perp \perp}[9,(3.3)]$, hence it is enough to show $\left(T^{t} \phi\right)(a)=0$ for every $a \in I^{\perp}$. Now $I^{\perp}$ is a closed ideal in $E$ $[9,(3.2)]$, hence $T I^{\perp} \subset I^{\perp}$; it follows that for $a \in I^{\perp},\left(T^{\prime} \phi\right)(a)=\phi(T a)=0$. We 
thus have that $T^{t}$ is a continuous multiplication operator on $\tilde{\mathbf{\Omega}}$. Our mapping is one-one, since the inverse mapping exists. It of course preserves the algebraic operations; and finally, from (6.1), it preserves order.

We return to our locally compact space $X$. For each $r \in C$, let us denote by $T_{r}$ the linear transformation of $C^{k}$ into itself defined by $\left(T_{r} f\right)(x)=r(x) f(x)$ for every $x \in X$. It is easily verified that $T_{r}$ carries each ideal in $C^{k}$ into itself, hence is a multiplication operator.

Now given a partially ordered vector space, the concepts $a \bigvee b$ and $a \wedge b$ are well-defined even though they may not always exist; a fortiori, the same holds for the concept of sub-vector-lattice. And similarly, given a partially ordered ring, the concept of sub-lattice-ordered-ring is well-defined.

(6.5) The mapping $r \rightarrow T_{r}$ is an imbedding of the lattice-ordered ring $C$ as a sub-lattice-ordered-ring of $B\left(C^{k}\right)$. The constant function $1(X)$ is carried by this imbedding into the identity operator.

Proof. Straightforward computation gives us that $T_{r+s}=T_{r}+T_{s}, T_{\lambda_{r}}=\lambda T_{r}$, $T_{r s}=T_{r} T_{s}$, and $T_{1(x)}$ is the identity operator. The mapping is one-one. For, suppose $r \neq 0$; this means $r(x) \neq 0$ for some $x \in X$; choosing $f \in C^{k}$ such that $f(x) \neq 0$, we have $\left(T_{r} f\right)(x) \neq 0$, whence $T_{r} f \neq 0$ and thus $T_{r} \neq 0$. It remains to show $T_{r \vee s}=T_{r} \vee T_{s}$. This follows easily from the following: given $f \in C^{k}+$ and $x \in X, \quad\left(T_{r \vee} f\right)(x)=(r \bigvee s)(x) f(x)=\max (r(x) f(x), s(x) f(x))=\max \left(\left(T_{r} f\right) \quad(x)\right.$, $(T, f)(x))$.

Henceforth we will identify $C$ with its image under the above mapping, and denote $T_{r}$ simply by $r$; that is, for each $f \in C^{k}, r f$ is the element of $C^{k}$ satisfying $(r f)(x)=r(x) f(x)$ for every $x \in X$. As is natural, the adjoint operator on $L^{k}$ will then be denoted by $r^{t}$.

(6.6) For each $r \in C, r^{t}$ is a continuous multiplication operator on $L^{k}$.

Proof. From the above isomorphism theorem, the operator $r$ is the difference of two positive operators. It follows from the corollary of (6.2) that $r^{t}$ is continuous. To complete the proof, it is enough to show that for each $\mu \in L^{k}$, $r^{t} \mu \in L^{k}{ }_{\mu}$. We assume (as we can) that $r \geqq 0$ and $\mu \geqq 0$, and we show precisely that $\left(r^{t} \mu\right)(f)=\left(r^{t} \mu\right)_{\mu}(f)$ for all $f \in C^{k}{ }_{+}$.

Now $\left(r^{i} \mu \wedge n \mu\right)(f) \leqq\left(r^{i} \mu\right)_{\mu}(f) \leqq\left(r^{\prime} \mu\right)(f)$. We claim that for $n$ sufficiently large, the first and last of these are actually equal. In effect

$$
\left(r^{i} \mu \wedge n \mu\right)(f)=\inf _{g, h \geq 0,0+h=f}\left(\left(r^{t} \mu\right)(g)+(n \mu)(h)\right)=\inf _{0, h \geqq 0,0+h=f}(r g+n h) .
$$

Since, for $n \geqq \sup _{r \in \text { support } f} r(x)$, we have $r g+n h \geqq r g+r h=r(g+h)=r f$, hence $\mu(r g+n h) \geqq \mu(r f)=\left(r^{i} \mu\right)(f)$, it follows that for such $n,\left(r^{i} \mu \wedge n \mu\right)(f) \geqq\left(r^{i} \mu\right)(f)$, and we are through.

(6.6) says that every closed ideal in $L^{k}$ is invariant under $r^{t}$. Since in particular, this holds for principal closed ideals, it follows easily that every $\sigma$-closed ideal is invariant under $r^{t}$. In particular, 
(6.7) For each $r \in C, r^{t}\left(\sigma L^{\omega}\right) \subset \sigma L^{\omega}$.

We consider the adjoint $r^{t t}$ of $r^{t}$. From (6.6) and (6.3), $M^{k}$ is invariant under $r^{t t}$; hence we can confine our attention to the restriction of $r^{t t}$ to $M^{k}$. Since the latter is the only part of $\Omega\left(L^{k}\right)$ with which we are concerned, we will henceforth use $r^{t t}$ to denote this restriction. It follows from (6.4) and (6.6) that $r^{t t}$ is a continuous multiplication operator on $M^{k}$. (Actually, from (d) in (7.1), every ideal-not just every closed ideal-in $M^{k}$ is invariant under $r^{t l}$.)

7. The Stone space of $M^{k}$. By the Stone space of $M^{k}$ we mean the locally compact space defined by $\mathrm{H}$. Bauer $[1 ; 2]$. Since, however, we are working with vector lattices, we will use the Kakutani representation theorem for $(M)$-spaces $[7 ; 12]$ rather than the Gelfand one for rings.

$C^{b}(Y)$ below denotes the set of bounded continuous functions on $Y$.

(7.1) There exists a locally compact space $Y$ with the following properties:

(a) $C^{b}(Y)=M^{\omega}$;

(b) $C^{k}(Y) \subset M^{k} \subset C^{\omega}(Y)$ (hence $C^{\omega}(Y)$ is the norm-closure of $M^{k}$ );

(c) $Y$ is hyperstonian [6], that is, it is extremely disconnected and $\dot{\Omega}\left(C^{k}(Y)\right)$ is separating on $C^{k}(Y)$;

(d) $C(Y)$ is precisely the set of continuous multiplication operators on $L^{k}$.

Most of the present section will be devoted to the proof of (7.1). We point out first, however, that in terms of (7.1) we can state the

(7.2) Fourth form of the Question: Do we have $M^{k}=C^{k}(Y)$ ? Otherwise stated, does every element of $M^{k}$ have compact support on $Y$ ?

Now let us denote by $m^{k}$ the set $\left\{f \in M^{k} \mid 1_{f} \in M^{k}\right\}$ ( $m^{k}$ is of course all of $M^{k}$ if the answer to our Question is yes (5.2)). We note first that $m^{k}$ is normdense in $M^{k}$; because for each $f \in M^{k}{ }_{+},(f-(1 / n) 1)^{+}$is in $m^{k}$ (cf. (5.1) and (5.3)) for $n=1,2, \cdots$, and clearly $\lim _{n}\|f-(f-(1 / n) 1)+\|=0$. It follows that the norm-closure of $m^{k}$ in $M^{\omega}$ is identical with that of $M^{k}$; we denote this norm-closure by $\mathbf{M}^{k}$.

(7.3) If a component e of 1 lies in $\mathbf{M}^{k}$, then it lies in $M^{k}$, hence in $m^{k}$.

Proof. There exists $f \in M^{k}$ such that $\|e-f\|<1 / 2$. Moreover we can take $f$ to satisfy $0 \leqq f \leqq e$, since $\left\|e-f^{+} \wedge e\right\| \leqq\|e-f\|$, and so we can replace $f$ by $f^{+} \wedge e$ if necessary. We now have $0 \leqq e-f \leqq(1 / 2) e$, whence $(1 / 2) e \leqq f$ and thus $e \in M^{k}$.

(7.4) Coroltaky. For every $f \in \mathbf{M}^{k}$ and $\lambda>0,(f-\lambda 1)+\in m^{k}$.

Proof. $1_{(f-\lambda)^{+}} \leqq(1 / \lambda)|f|$ (cf. (ii) in the proof of $[9,(6.1)]$ ), hence is in $\mathbf{M}^{k}$. Now apply (7.3).

(7.5) $\mathbf{M}^{k}+R 1$ is a sub-vector-lattice of $M^{\omega}$ and is norm-closed. Thus it is an $(M)$-space with 1 for strong order unit. 
Proof. To prove $\mathbf{M}^{k}+R \mathbf{1}$ is a sub-vector-lattice, it is enough to show that given $f+\lambda 1 \in \mathbf{M}^{k}+R 1$, we have $(f+\lambda 1)^{+} \in \mathbf{M}^{k}+R 1$ also. If $\lambda<0$, then $(f+\lambda 1)^{+}$is in $m^{k}$ by (7.4), and we are through. If $\lambda \geqq 0$, then $(f+\lambda 1)^{-}$ $=(-f-\lambda 1)^{+} \in m^{k}$, and we have $(f+\lambda 1)^{+}=f+\lambda 1-(f+\lambda 1)^{-}=\left[f-(f+\lambda 1)^{-}\right]$ $+\lambda 1 \in M^{k}+R 1$. It remains to show $\mathbf{M}^{k}+R 1$ is norm-closed in $M^{\omega}$. If $1 \in \mathbf{M}^{k}$, then $\mathbf{M}^{k}=M^{\omega}$ and we are through; hence we can assume $1 \in \mathbf{M}^{k}$.

LEMma. Given $f+\lambda 1, f \in \mathbf{M}^{k}$, if $\|f+\lambda 1\| \leqq n$, then $|\lambda| \leqq 2 n$.

The inequality on the norm gives us $f+\lambda 1 \geqq-n 1$. Writing $f=f \wedge n 1$ $+(f-n 1)^{+}$, this gives $(f-n 1)^{+} \geqq-\lambda 1-f \wedge n 1-n 1 \geqq-\lambda 1-n 1$ $-n \mathbf{1}=(-\lambda-2 n) 1$. If we had $\lambda \leqq-2 n$, this would give us $1 \in \mathbf{M}^{k}$ (by (7.4) again), contradicting our assumption. Thus $\lambda \geqq-2 n$. Writing the inequality on the norm in the form $-f+(-\lambda) 1 \geqq-n 1$, the above argument gives us that $-\lambda \geqq-2 n$ or $\lambda \leqq 2 n$. Thus $|\lambda| \leqq 2 n$, and the lemma is proved.

Now suppose $f \in M^{\omega}$ is in the norm-closure of $\mathbf{M}^{k}+R 1$. Then we have a sequence $\left\{f_{j}+\lambda_{j} 1\right\}$ of elements of $\mathbf{M}^{k}+R 1$ such that $\lim _{j}\left\|f_{j}+\lambda_{j} 1-f\right\|=0$. Writing $\left\|f_{j}+\lambda_{j} 1\right\| \leqq\left\|f_{j}+\lambda_{j} 1-f\right\|+\|f\|$, the convergence gives us that the set $\left\{f_{j}+\lambda_{j} 1\right\}$ is norm-bounded, hence from the lemma, the set of real numbers $\left\{\lambda_{j}\right\}$ is bounded too. Thus, by taking a subsequence if necessary, we can assume the sequence $\left\{\lambda_{j}\right\}$ converges to some $\lambda$. It follows easily that $\lim _{j}\left\|f_{j}-(f-\lambda 1)\right\|=0$, whence $f-\lambda 1 \in \mathbf{M}^{k}$. Then $f=(f-\lambda 1)+\lambda 1 \in \mathbf{M}^{k}+R 1$, and we are through.

Applying the Kakutani theorem to $\mathbf{M}^{k}+R 1$, it can be identified with the Banach lattice $C(Z)$ for some compact space $Z$. If $1 \notin \mathbf{M}^{k}, \mathbf{M}^{k}$ is a maximal ideal in $C(Z)$, hence is the set of continuous functions on $Z$ which vanish at some fixed $\bar{y} \in Z$. We denote the complement of $\bar{y}$ by $Y$. If, however, $1 \in \mathbf{M}^{k}$, then $\mathbf{M}^{k}=C(Z)$, and we take $Y=Z$. We show $Y$ is the space of (7.1).

From the definition of $Y$, we of course have that $\mathbf{M}^{k}=C^{\omega}(Y)$. Since $\mathbf{M}^{k}$ is a complete vector lattice, it follows $Y$ is extremely disconnected. We now show $m^{k}=C^{k}(Y)$. We can assume $1 \notin m^{k}$ (else there is nothing to prove). Consider $f \in m^{k}$. By definition of $m^{k}, 1_{f} \in m^{k}$ also, hence $1_{f} \in C^{\omega}(Y)$ and thus vanishes at infinity. In particular the set $\left\{y \in Y \mid 1_{f}(y) \geqq 1\right\}$ is compact. Since $1_{f}$ has only values 0,1 on $Y\left(M^{k}+R 1=C(Z)\right)$, this set is the support of $1_{f}$, hence of $f$. Thus $m^{k} \subset C^{k}(Y)$. Conversely, consider $f \in C^{k}(Y)$. Since $Y$ is extremely disconnected, $1_{f}$ has the same support as $f$, hence is in $C^{k}(Y)$ also. It follows from (7.3) that $1_{f} \in m^{k}$.

With our results so far, we have (b) of (7.1). We next show (a). Let us denote the isomorphic mapping of $\mathbf{M}^{k}$ onto $C^{\omega}(Y)$ by $p$, and for the present we will distinguish between $f \in \mathbf{M}^{k}$ and its image $p f$. We extend $p$ to an isomorphism of $M^{\omega}$ onto $C^{b}(Y)$. We define $p 1$ to be the function $1(Y)$ of constant value 1 on $Y$. Now consider $f \in M^{\omega}$ with, say, $0 \leqq f \leqq n 1$, and let $A_{f}$ $=\left\{g \in M^{k} \mid 0 \leqq g \leqq f\right\} . p A_{f}$ is bounded above by $n 1(Y)$, hence has a supremum in $C^{b}(Y)$; set $p f$ equal to this supremum. We have thus extended $p$ to a map- 
ping of $M^{\omega}+$ into $C^{b}(Y)_{+}$. This extension is clearly order-preserving and oneone. We show it is onto. Consider $h \in C^{b}(Y)$, with, say, $0 \leqq h \leqq n 1(Y)$, and let $A=\left\{g \in M^{k} \mid 0 \leqq p g \leqq h\right\}$. Since $p M^{k} \supset C^{k}(Y)$ (and $Y$ is locally compact); $h=\mathrm{V} p A$ in $C^{b}\left(Y^{z}\right)$. Now $A$ is bounded above in $M^{\omega}$ by $n 1$, hence has a supremum $f$ in $M^{\omega}$. We show $A=A_{f}$, whence it will follow $p f=h$. Consider $l \in A_{f}$. The set $\{g \wedge l \mid g \in A\}$ is contained in $A$ and $l$ is its supremum. It follows $p l=\mathrm{V}_{o \in A} p(g \wedge l) \leqq h$, hence $l \in A$.

We show $\tilde{\Omega}\left(C^{k}(Y)\right)$ is separating on $C^{k}(Y)$, which will establish (c) in (7.1). For each $K$ in $X, M(K) \subset m^{k}$, and as we have seen, $m^{k}=C^{k}(Y)$. We thus have $U_{\mathrm{all} K}$ in $x M(K) \subset C^{k}(Y) \subset M^{k}$. From (4.4), the first and last of these have the same $\tilde{\Omega}$, viz. $L^{k}$; it follows that also $\tilde{\Omega}\left(C^{k}(Y)\right)=L^{k}$. Since $L^{k}$ is separating on $M^{k}$, we are through.

It remains to prove (d) in (7.1), so we proceed to examine $C(Y)$ as a ring of operators on $L^{k}$. We note first that since $Y$ is extremely disconnected, $C(Y)$ is a complete $f$-ring [3]. Each $r \in C(Y)$ is an operator on $C^{k}(Y)$ under $(r f)(y)=r(y) f(y)$. Moreover, it is easily verified that $r$ is a continuous multiplication operator on $C^{k}(Y)$. We denote the adjoint operator in $L^{k}$ by $r^{t}$ (remember that $L^{k}$ is $L^{k}(X)$ not $L^{k}(Y)$; as we showed above, $L^{k}=\tilde{\Omega}\left(C^{k}(Y)\right.$ ) (cf. (6.3)), and the adjoint of $r^{t}$ in $M^{k}$ by $r^{t t}$.

(7.6) $r^{t t}$ coincides with $r$ on $M^{k}$, that is, $r^{t} f=r f$ for every $f \in M^{k}$.

Proof. We show first that the theorem holds for $f \in C^{k}(Y)$. For every $\mu \in L^{k}, \mu\left(r^{t} f\right)=\left(r^{t} \mu\right)(f)=\mu(r f)$. Since $L^{k}$ is separating on $C^{k}(Y)$, it follows $r^{t} f=r f$. In more detail $\left(r^{t} f\right)(y)=r(y) f(y)$ for all $y \in Y$. Now consider $f \in M^{k}$. We can clearly confine ourselves to the case where $f \geqq 0, r \geqq 0$. For each $n=1,2, \cdots$, denote $(f-(1 / n) 1)^{+}$by $g_{n}$. Then $g_{n} \in C^{k}(Y)$ (7.4), hence $r^{t t} g_{n}=r g_{n}$. Now $g_{n} \uparrow f$; in fact the convergence is norm-convergence, hence pointwise. It follows on the one hand from (6.2) that $r^{t t} g_{n} \uparrow r^{t t} f$, and on the other that $\left(r^{\prime \prime} g_{n}\right)(y)=r(y) g_{n}(y) \uparrow r(y) f(y)$ for every $y$. Since this latter function is continuous, it is the supremum of the $r^{t t} g_{n}$ 's, hence is precisely $r^{t} f$. In short, $\left(r^{t} f\right)(y)=r(y) f(y)$ for every $y$.

(7.7) Corollary. For every $r \in C(Y)$ and $f \in M^{k}$, the function defined by $r(y) f(y)$ is also in $M^{k}$; thus $C(Y)$ is a ring of continuous multiplication operators on $M^{k}$.

REMARK. If we knew that $M^{k}=C^{k}(Y)$, this would be trivial. In the absence of such knowledge, the corollary states a strong property of $Y$, since for a general locally compact space $X, C^{k}$ is the largest subspace of $C^{b}$ which $C$ can carry into itself (cf. (7.8)).

We turn to the proof of (d). From (6.4), it is enough to show that $C(Y)$ is the ring of continuous multiplication operators on $M^{k}$. The above corollary gives us half of this, viz. that $C(Y)$ is contained in this ring. Since the ring is commutative (cf. the discussion in $\$ 6$ ), it follows in particular that each 
element of $C(Y)$ commutes (as an operator) with every continuous multiplication operator on $M^{k}$. We now show every continuous multiplication operator on $M^{k}$ is given by an element of $C(Y)$.

Consider first the case where $X$ is compact, hence $M^{k}=M=C(Y)$, and let $T$ be a continuous multiplication operator on $M$. Denote $T 1$ by $g$. We claim that for every $f \in M,(T f)(y)=g(y) f(y)$ for all $y$. In effect, $T f=T(f \cdot 1)=f(T 1)$ (by the commutativity pointed out above) $=f g$. Now consider the general case of $X$ locally compact. In the following, the letter $K$ will always denote a compact set in $Y$ which is the closure of its interior, whence $1(K) \in M^{k}$. Let $T$ be a continuous multiplication operator on $M^{k}$. For each $K$, denote $T 1(K)$ by $g_{K}$. Then for every $f \in M^{k}$ with support in $K, T f=g_{K} f$, by the preceding argument. Now it is easily verified that for every pair $K_{1}, K_{2}, g_{K_{1}}$ and $g_{K_{2}}$ coincide on $K_{1} \cap K_{2}$. It follows we have a well-defined element $r$ of $C(Y)$ which coincides on each $K$ with $g_{K}$. We claim that for every $f \in C^{k}(Y), T f=r f$. In effect, letting $K$ be the support of $f, T f=g_{K} f=r f$. Applying (7.6) gives us that $T f=r f$ for every $f \in M^{k}$, and completes the proof of (7.1).

Since $C^{k} \subset \mathrm{U}_{\mathrm{all} K}$ in $\mathrm{x} M(K) \subset C^{k}(Y)$, the support in $Y$ of an element of $C^{k}$ is always compact. As we have stated, we do not know whether the support in $Y$ of an element of $M^{k}$ need be compact; and indeed, this is our fourth form of the Question. We can however show the following, which is essentially due to Mack [10, Proposition 2.3.11]. (A topological space is pseudocompact if every continuous function on it is bounded).

(7.8) The support in $Y$ of every element of $M^{k}$ is pseudocompact.

This theorem follows from (7.7) and the

LEMma. Let $W$ be a topological space, $f$ a bounded real function on $W$, and suppose that for every $r \in C(W), r(w) f(w)$ defines a bounded function. Then the support $F$ of $f$ is pseudocompact.

Proof. Suppose there exists $r \in C(W)$ which is unbounded on $F$; we can take $r$ strictly positive. Since the set $G=\{w \mid f(w) \neq 0\}$ is dense in $F, r$ is unbounded in $G$; hence there exists a sequence $\left\{w_{n}\right\} \subset G$ such that $\left\{r\left(w_{n}\right)\right\}$ increases monotonically to $+\infty$. Let $u$ be the continuous real function on $R$ defined by $u(\lambda)=0$ for $\lambda \leqq 0, u\left(r\left(w_{n}\right)\right)=n / f\left(w_{n}\right)$ for $n=1,2, \cdots$, and $u(\lambda)$ is given elsewhere by the straight line segments between the successive values at $0, r\left(w_{1}\right), r\left(w_{2}\right), \cdots$. Since $u$ is continuous, the function $s$ on $W$ defined by $s(w)=u(r(w))$ is in $C(W)$; hence by hypothesis, the function $s(w) f(w)$ is bounded. But $s\left(w_{n}\right) f\left(w_{n}\right)=n$, which gives a contradiction.

We can now show that the second form of the Question (5.4) is actually equivalent to the others. Assume (5.4) holds for every element of $M^{k}$. Consider $f \in M^{k}{ }_{+}$and denote its support in $Y$ by $F$. Let $g$ be an element of $M^{k}+$ given by (5.4). It will be enough to show that $1(F)$, the characteristic function of $F$ on $Y$, is dominated by some multiple of $g$. We show first that $g(y)>0$ 
for every $y \in F$. Given such a $y$, let $H$ be an open compact neighborhood of $y$ contained in $F$. Then $\mathrm{V}_{n} 1(H) \wedge n f=1(H)$, hence $1(H) \in M_{f}^{k}$, hence $1(H)$ is dominated by some multiple of $g$. In particular, $g(y)>0$. Now $F$ is pseudocompact (7.8), hence $g$ attains its infimum on $F$. It follows $g(y) \geqq \lambda>0$ on all of $F$, whence $1(F) \leqq(1 / \lambda) g$.

REMARK. $C$ is imbedded in $C(Y)$ by the mapping $r \rightarrow r^{t t}$. Since $C^{k}$ can be considered a subring of $C$, it is also imbedded in $C(Y)$ by this mapping. Now we also have the natural imbedding of $C^{k}$ (as a sub-vector-lattice) in $C^{k}(Y)$ $C C(Y)$. It is not hard to show that these two imbeddings are identical.

\section{APPENDIX}

8. More on operators. We note first the easily verified fact that a positive multiplication operator on a vector lattice $E$ preserves the lattice operations on $E$.

In $\$ 6$ we discussed some properties of $C$ as a ring of operators on $C^{k}, L^{k}$, and $M^{k}$. Given an element $r$ of $C, r C^{k}$ need not in general be even a sub-vectorlattice of $C^{k}$. If $r \geqq 0$, then from the above remark, we do have that $r C^{k}$ is a sub-vector-lattice; however, it need not be an ideal. In $L^{k}$ the situation is better. Specifically, our aim in this section is to prove

(8.1) Given any $r \in C, r^{t} L^{k}$ is an ideal in $L^{k}$.

We will prove this after some preliminary material (cf. $[10, \S 2.1])$. Let $r$ be an element of $C$ and $I$ the ideal in $C^{k}$ generated by $r C^{k}$. Then $r^{t} I^{\perp}=0$ (general property of the adjoint operator). Writing $L^{k}=I^{\perp} \oplus I^{\perp \prime}$, it follows from the fact that $r^{t}$ is a multiplication operator (6.6), that $r^{t} L^{k} \subset I^{\perp \prime}$.

(8.2) Given $r \in C, s \in C$, if $|r| \wedge|s|=0$, then $r^{t} L^{k}$ and $s^{t} L^{k}$ are disjoint.

Proof. Let $I, J$ be the ideals in $C^{k}$ generated by $r C^{k}, s C^{k}$ respectively. It is clear from the pointwise multiplication that $I \cap J=0$. Then $I^{\perp \prime} \cap J^{\perp \prime}=0$, $r^{t} L^{k} \subset I^{\perp \prime}$, and $s^{t} L^{k} \subset J^{\perp \prime}$.

(8.3) The mapping $r \rightarrow r^{t}$ is an isomorphism of the lattice-ordered ring $C$ into $\mathrm{B}\left(L^{k}\right)$.

Proof. The only property requiring proof (cf. (6.5)) is that the mapping preserves the lattice operations. It is enough to show that $\left(r^{+}\right)^{t}=\left(r^{t}\right)^{+}$. Now $\left(r^{+}\right)^{t} \wedge\left(r^{-}\right)^{t}=0$ : for, suppose $T \in B\left(L^{k}\right), 0 \leqq T \leqq\left(r^{+}\right)^{t}, 0 \leqq T \leqq\left(r^{-}\right)^{t}$; applying (8.2), we obtain $T=0$. Since $r^{t}=\left(r^{+}-r^{-}\right)^{t}=\left(r^{+}\right)^{t}-\left(r^{-}\right)^{t}$, this gives the required identity.

(8.4) Given $r \in C, r^{t} L^{k}=|r|^{t} L^{k}$.

Proof. Consider $\mu \in L^{k}$; we show there exists $\nu \in L^{k}$ such that $|r|^{t} \nu=r^{t} \mu$ and $r^{i} \nu=|r|^{i} \mu$. Let $I$ be the closed ideal in $L^{k}$ generated by $\left(r^{+}\right)^{t} L^{k}$, and set $J=I^{\prime}$. It follows $\left(r^{+}\right)^{t} J=0=\left(r^{-}\right)^{t} I$ (from (8.2) and (6.6)). Then $\mu_{I}-\mu_{J}$ is the 
required $\nu$. For, $|r|^{i} \nu=|r|{ }^{i} \mu_{I}-|r|^{i} \mu_{J}=\left(r^{+}\right)^{t} \mu_{I}-\left(r^{-}\right)^{t} \mu_{J}=r^{i} \mu_{\mu}+r^{i} \mu_{J}=r^{i} \mu_{\text {; }}$ and similarly for $r^{t} \nu$.

We proceed to prove (8.1). From (8.4), we can take $r \geqq 0$ (hence $r^{t} L^{k}$ is a sub-vector-lattice). Let us denote $\left(r-n^{-1} 1(X)\right)+$ by $r_{n}, n=1,2, \cdots$. Then for every $\mu \in L^{k}{ }_{+}, r_{n}^{t} \mu \uparrow r^{i} \mu$. In effect, $\lim _{n}\left\|r-r_{n}\right\|=0$, hence for each $f \in C^{k}{ }_{+}$, $\lim _{n}\left\|r f-r_{n} f\right\|=0$, hence in turn, $\lim _{n} \mu\left(r_{n} f\right)=\mu(r f)$; this says $\lim _{n}\left(r_{n}^{i} \mu\right)(f)$ $=\left(r^{t} \mu\right)(f)$, and since $\mu \geqq 0$, the convergence is monotonic.

Let $I_{n}$ be the closed ideal in $L^{k}$ generated by $r_{n}^{t} L^{k}$. We show

(i) $r^{t}$ carries each $I_{n}$ onto itself isomorphically.

We do this by showing that $r^{t}$ coincides on $I_{n}$ with the operator $\left[\left(n^{-1} 1(X)\right) \vee r\right]^{t}$, which has an inverse. Consider $\mu \in I_{n} . r^{t} \mu=\left(n^{-1} 1(X)\right)^{t} \mu$ $+\left(r-n^{-1} 1(X)\right)^{t} \mu=\left(n^{-1} 1(X)\right)^{t} \mu+\left[\left(r-n^{-1} 1(X)\right)^{+}\right]^{t} \mu$ (definition of $I_{n}$; cf. the argument in $(8.4))=\left[n^{-1} 1(X)+\left(r-n^{-1} 1(X)\right)+\right]^{t} \mu=\left[\left(n^{-1} 1(X)\right) \bigvee_{r}\right]^{t} \mu$.

(i) gives us that $r^{t} L^{k}$ contains every $I_{n}$, hence the ideal $U_{n} I_{n}$; moreover, from the preceding paragraph, this ideal is dense in $r^{t} L^{k}$. It follows it is dense in the closed ideal generated by $r^{t} L^{k}$. Hence

(ii) if $\nu \geqq 0$ is in the closed ideal generated by $r^{t} L^{k}$, then $\nu_{I_{n}} \uparrow \nu$.

To complete the proof, it is enough, since $r^{t} L^{k}$ is a sub-vector-lattice, to show the following: if $0 \leqq \nu \leqq r^{t} \mu$, there exists $\rho \in L^{k}$ such that $r^{t} \rho=\nu$. For simplicity, denote $\nu_{I_{n}}$ by $\nu_{n}, n=1,2, \cdots$. By (i), for each $n$ there exists $\rho_{n} \in\left(I_{n}\right)_{+}$such that $r^{t} \rho_{n}=\nu_{n}$. Moreover $\rho_{n} \leqq \mu$ : for, $r^{t}\left(\rho_{n} \wedge \mu\right)=r^{t} \rho_{n} \wedge r^{t} \mu$ $=\nu_{n} \wedge r^{t} \mu=\nu_{n}$ (since $\left.\nu_{n} \leqq \nu \leqq r^{t} \mu\right)$; since $r^{t}$ is one-one on $I_{n}$, we must thus have $\rho_{n}=\rho_{n} \wedge \mu$. Thus the $\rho_{n}$ 's are bounded above; denote $V_{n} \rho_{n}$ by $\rho$. Since $r^{t}$ is an isomorphism on $I_{n+1}, \rho_{n} \leqq \rho_{n+1}$ for all $n$; hence $\rho_{n} \uparrow \rho$. Since finally, $r^{t}$ is continuous on $L^{k}$ and $\nu_{n} \uparrow \nu$ (from (ii) above), we have $r^{t} \rho=\nu$.

9. The Radon-Nikodym theorem. For a fixed $\mu<L^{k}$, the mapping $r \rightarrow r^{t} \mu$ is a linear mapping of $C$ (as a vector space) into $L^{k}{ }_{\mu}$, with $1(X)$ being mapped onto $\mu$. We denote this mapping by $\mu: C \rightarrow L^{k}{ }_{\mu}$, that is, $\mu r=r^{t} \mu$. If $\mu \geqq 0$, the mapping is a vector lattice homomorphism. To prove this, it is enough to show that $\mu r^{+}=(\mu r)^{+}$. Well, $\mu r=\mu r^{+}-\mu r^{-}=\left(r^{+}\right)^{t} \mu-\left(r^{-}\right)^{t} \mu$; since these are disjoint by (8.2), the required identity follows.

Our object in this section is to prove

(9.1) If $\mu \geqq 0, \overline{\mu C}=L^{k}{ }_{\mu}$.

From $[9,(3.6)]$ it is enough to prove

(9.2) If $\mu \geqq 0, \mu C$ is separating on $M^{k}{ }_{\mu}$.

Proof. Consider $f \in M^{k}{ }_{\mu}, f \neq 0$; we can take $|f| \leqq 1$. We have to show there exists $r \in C$ such that $(\mu r)(f) \neq 0$. If $f>0$ or $f<0$, we are through, since then $\mu(f) \neq 0$ and therefore $1(X)$ is the required $r$. Suppose $f^{+} \neq 0$ and $f^{-} \neq 0$. From (3.7) there exists $g \in C^{k}$ such that $g \wedge f^{+}>0$. Since $g f^{+}=\left(g \wedge f^{+}\right)\left(g \vee f^{+}\right)$, it follows $g f^{+}>0$ also, hence $\mu\left(g f^{+}\right)>0$.

Write $L^{k}{ }_{\mu}=I \oplus J$, where $\nu\left(f^{+}\right)=0$ for all $\nu \in J$ and $\nu\left(f^{-}\right)=0$ for all $\nu \in I$ 
$[9,(3.4)]$ (to correct a misprint: $\Omega$ in that theorem should be $\tilde{\Omega}$ ), and choose $\epsilon<\mu\left(g f^{+}\right) / 2$. There exist $h \in C^{k}{ }_{+}, k \in C^{k}{ }_{+}$such that $h+k=g$ and $\mu_{I}(k)+\mu_{J}(h)$ $<\epsilon\left[4\right.$, Chapter II, $\delta 2$, Proposition 3]. We show $\mu(h f)>0 ;$ since $\mu(h f)=\left(h^{i} \mu\right)(f)$ $=(\mu h)(f)$, this will complete the proof.

Now $\mu\left(h f^{+}\right)=\mu\left(g f^{+}-k f^{+}\right)=\mu\left(g f^{+}\right)-\mu\left(k f^{+}\right)=\mu\left(g f^{+}\right)-\mu_{I}\left(k f^{+}\right) \geqq \mu\left(g f^{+}\right)$ $-\mu_{I}(k)$ (since $\left.|f| \leqq 1\right)>\mu\left(g f^{+}\right)-\epsilon$; and $\mu\left(h f^{-}\right)=\mu_{J}\left(h f^{-}\right) \leqq \mu_{J}(h)<\epsilon$. Thus $\mu(h f)$ $=\mu\left(h f^{+}\right)-\mu\left(h f^{-}\right)>\mu\left(g f^{+}\right)-2 \epsilon>0$.

\section{BIBLIOGRAPHY}

1. H. Bauer, Über die Beziehungen einer abstrakten Theorie des Riemann-Integrals zur Theorie Radonscher Masse, Math. Z. vol. 65 (1956) pp. 448-482.

2. - Sur l'equivalence des theories de l'integration selon N. Bourbaki et selon M. H. Stone, Bull. Soc. Math. France vol. 85 (1957) pp. 51-75.

3. G. Birkhoff and R. S. Pierce, Lattice-ordered rings, An. Acad. Brasil. Ci. vol. 28 (1956) pp. 41-69.

4. N. Bourbaki, Integration, Paris, Hermann et Cie., 1952, Chapters I-IV.

5. - Integration, Paris, Hermann et Cie., 1956, Chapter V.

6. J. Dixmier, Sur certains espaces consideres par M. H. Stone, Summa Brasil. Math. vol. 2 (1951) pp. 151-182.

7. S. Kakutani, Concrete representation of abstract (M)-spaces, Ann. of Math. vol. 42 (1941) pp. 994-1024.

8. S. Kaplan, On the second dual of the space of continuous functions, Trans. Amer. Math. Soc. vol. 86 (1957) pp. 70-90.

9. - The second dual of the space of continuous functions. II, Trans. Amer. Math. Soc. vol. 93 (1959) pp. 329-350.

10. J. E. Mack, The order dual of the space of Radon measures, Purdue Dissertation, 1959.

11. H. Nakano, Modern spectral theory, Tokyo, Maruzen Co., 1950.

12. K. Yosida, On vector lattice with a unit, Proc. Japan Acad. vol. 17 (1941) pp. 121-124.

Wayne State University,

Detroit, Michigan 